\title{
Les résistances au travail en perspective
}

\author{
Mécaniciens et caissières en seconde partie de \\ carrière \\ Morgane Kuehni \\ Professeure à la Haute école de travail social et de la santé, \\ École d'études sociales et pédagogique (ÉÉSP) de Lausanne
}

(Suisse)

[Résumé] Cet article interroge les effets du vieillissement sur les résistances au travail des salarié.e.s de deux groupes professionnels: caissières et mécaniciens sur locomotive. Basé sur une recherche empirique, le propos vise moins à circonscrire les formes de résistance, que questionner leur pluralité et explorer les enjeux sexués et les ressources différenciées qui les sous-tendent.

Mots-clés : vieillissement actif, seniors, genre, résistances au travail

[Abstract] This paper analyzes the aging effects on work resistance among female and workers in two professional groups : cashiers and train engineers. Based on field work data, it aims more at defining forms of resistance to question their plurality and explore the gendered stakes and different ressources they underpin.

Keywords : Active ageing, seniors, gender, resistance at work

All quiet on the workplace front ? 》 La question soulevée en 1995 par Thompson et Ackroyd dans un contexte de mutations profondes du marché du travail a trouvé depuis quelques années un regain d'intérêt chez les sociologues du travail francophones (Bélanger et Thuderoz 2010 ; Bouquin 2008; Rot 2000). Grèves, lock-out, manifestations, autant de résistances pratiquées durant la période industrielle fordiste, semblent aujourd'hui perdre du terrain avec l'affaiblissement du syndicalisme, la précarisation de l'emploi et du travail (Bouffartigue 2008). La "montée des incertitudes» (Castel 2009), les nouvelles formes d'organisation du travail et de management n'ont pourtant pas eu raison de toute forme de conflictualité sociale: les mondes du travail demeurent des «territoires de contestation ». Les enquêtes de terrain montrent que les mobilisations collectives, pétitions, débrayages et grèves sont toujours d'actualité même chez les salariée.es les plus précaires (Puech 2004), mais aussi qu'ils et elles nourrissent des formes d' « esprit de révolte sans révolte » (Zarifian 2004) et de " contestations silencieuses » (Jounin 2008). Cette pluralité des registres de contestation s'explique non seulement par les modalités des relations professionnelles et le jeu des rapports de force qui les structurent, mais fait également écho aux conditions de travail et aux parcours professionnels et biographiques des travailleurs/euses. La majorité des auteurs s'entendent sur la nécessité d'une approche contextualisée des résistances, mais les débats sont vifs sur les manières de les définir et 
de les problématiser. Les résistances s'expriment-elles forcément sur un registre d'opposition frontal ou peuvent-elles éclore à l'abri des regards, au travers des aménagements quotidiens destinés à se préserver et se réapproprier une partie de son travail ? Ces pratiques clandestines contribuent-elles à atténuer ou à renforcer les rapports de domination? Autant de questions qui esquissent aujourd'hui les grandes lignes d'un champ de recherche en chantier et voué à le rester, puisque les résistances s'élaborent toujours en imbrication avec les situations de travail, son organisation et ses transformations.

Cet article entend participer au débat en focalisant l'attention sur une question spécifique : le vieillissement joue-t-il un rôle sur les possibilités ou les capacités de résistances au travail des salarié.e.s ? Si la question s'avère d'une grande actualité dans un contexte d'encouragement étatique au "vieillissement actif » particulièrement saillant en Suisse, elle est rarement traitée. Loin d'apporter une réponse unilatérale, cet article propose une mise en perspective des formes de résistances de deux groupes professionnels : les mécaniciens sur locomotive et les caissières d'un grand magasin en seconde partie de carrière (50 ans et plus). Partant d'une recherche empirique, il s'agira moins de répertorier les stratégies de contestation que d'explorer les enjeux qui les sous-tendent et les jeux de rapports de force qui les structurent.

La première partie montre que, dans un contexte de pression étatique au vieillissement actif, les seniors ${ }^{1}$ doivent faire face à une double injonction: se maintenir en emploi le plus longtemps possible pour s'assurer des conditions de vie décente à la retraite et s'adapter aux changements de leur poste de travail, particulièrement aux pressions temporelles et exigences de rentabilité, qui s'accommodent mal de l'avancée en âge. La seconde partie s'arrête sur les marges de manœuvre différenciées des mécaniciens et des caissières pour répondre à cette injonction. Mobilisant une définition large des résistances au travail, comme une volonté pour les salarié.e.s « d’atténuer l'exploitation ou de desserrer l'étau du contrôle managérial » (Bouquin 2011: 70), il s'agira de montrer que les possibilités de résistance et les registres dans lesquels elle s'exprime se comprennent à la lumière des rapports sociaux où s'entremêlent à la fois objectivement et subjectivement les contraintes de la situation de travail et les ressources à disposition: capital retraite, soutien institutionnel dans et hors de l'entreprise.

Cet article s'appuie sur le matériau empirique recueilli dans le cadre de la recherche « EGALISE » 2 (Égalité des seniors en emploi) et mobilise des entretiens semi-directifs

1 Par commodité de langage, nous utiliserons le terme seniors pour désigner toutes les personnes actives de 50 ans et plus.

2 La recherche «EGALISE » (Égalité des seniors en emploi) est financée par le Programme national de recherche "Égalité entre hommes et femmes » du Fonds national suisse (PNR 60), dirigée par Nicky Le Feuvre et menée avec Magdalena Rosende et Céline Schoeni que 
réalisés dans trois grandes entreprises suisses du secteur tertiaire : une entreprise des transports et deux du commerce. Les secteurs d'activité investigués se distinguent par la composition diversifiée de leur main d'œuvre : l'entreprise de transport est à majorité masculine (85\%) et celles du commerce sont à majorité féminine (65\%). Cette contribution s'appuie sur une analyse de 24 entretiens menés avec des responsables des ressources humaines, des mécaniciens et des caissières ${ }^{3}$ de plus de 50 ans. Notons que les personnes enquêtées ont été sélectionnées par la hiérarchie et que nous n'avons pas eu accès aux seniors qui ont quitté l'entreprise suite à un licenciement, des problèmes de santé ou par choix. Nos enquêté.e.s cumulent une ancienneté importante au sein de leur entreprise, sont majoritairement d'origine suisse ou établi.e.s de longue date dans ce pays.

\section{Travail et vieillissement}

Dans un contexte d'encouragement au vieillissement actif fortement développé dans les pays industrialisés, la Suisse fait figure de «bonne élève » avec un taux d'activité de 70,5\% chez les 55-64 ans. Elle fait partie des rares pays où la majorité des actifs/ves

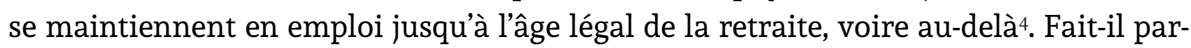
ticulièrement bon vieillir au travail dans ce pays?

\subsection{Contrainte au maintien en emploi}

Mentionnons d'emblée qu'en comparaison des travailleurs/euses d'autres classes d'âge, les seniors connaissent un faible taux de chômage et une forte stabilité professionnelle. En 2010, le taux de chômage s'élève à 3,5 \% chez les personnes actives de 55 à 64 ans $^{5}$ et le taux de rotation par âge met en évidence la relation décroissante entre

je remercie chaleureusement pour leur lecture avisée. Pour cet article, seule une partie des données a été utilisée.

3 Les extraits cités sont issus d'entretiens menés avec des caissières, toutefois l'analyse s'appuie sur un matériau plus large : plusieurs vendeuses interviewées ont fait des années de caisse avant d'être déplacées à leur demande pour des raisons de pénibilité du travail. 4 Cinq ans après l'âge légal de la retraite, $15 \%$ des hommes et $10 \%$ des femmes exercent encore une activité professionnelle (OFS 2011).

5 À titre de comparaison, le taux de chômage des $25-54$ est de $4,2 \%$. Notons que les chiffres disponibles pour le chômage des plus de 50 ans sont à manipuler avec une extrême prudence. D'une part, certaines personnes qui perdent leur emploi à cet âge rejoignent le rang des inactives plutôt que celles des chômeuses, et d'autre part plus de la moitié des seniors au chômage (53,1 \% en 2010) sont au chômage de longue durée, soit depuis plus d'une année (OFS 2011). 
l'âge et la mobilité professionnelle ${ }^{6}$. Les seniors sont-ils particulièrement satisfaits des postes occupés ou craignent-ils de se retrouver au chômage s'ils décidaient de changer d'employeur?

Au même âge, la position dans l'emploi et les perspectives de carrière sont très différentes selon le métier exercé et la position professionnelle, toutefois la majorité des études offrent une description plutôt alarmante de la situation des seniors sur le marché du travail dans les pays occidentaux : différents phénomènes de « dépréciation des salariés âgés » émergent (Guillemard 2003), les avantages liés à l'ancienneté se raréfient et les solidarités intergénérationnelles s'amenuisent (OCDE 2006). Les enquêtes statistiques disponibles, notamment l'enquête SHARE (Survey of Health, Ageing and Retirement in Europe) brosse un tableau plutôt sombre de la situation des seniors sur le marché du travail helvétique. En 2006, 54,9\% des travailleuses et travailleurs de plus de 50 ans déclarent travailler sous pression, 19,8\% estiment avoir peu de chance de garder leur emploi et $9 \%$ redoutent que leur état de santé limite leur capacité au travail7. Le fort sentiment d'insécurité professionnelle mis en exergue par cette enquête fait clairement écho à la faiblesse de couverture du risque de non-travail dans le système de protection sociale helvétique. Les mesures spécifiques pour les travailleurs/euses de plus de 50 ans et les politiques d'intégration dans l'emploi des seniors sont à ce jour très peu développées, alors même que le système de retraite pénalise lourdement les personnes qui quittent le marché du travail avant l'âge légal de la retraite fixé à 65 ans pour les hommes et 64 ans pour les femmes 8 . En effet, prendre une retraite anticipée équivaut à une diminution de la rente vieillesse de l'ordre de $6,8 \%$ par année d'anticipation. Plusieurs personnes se retrouvent donc contraintes au maintien en emploi pour des raisons économiques.

Notre enquête empirique confirme la nécessité de se maintenir en emploi pour des raisons financières, particulièrement pour les personnes qui vivent seules et/ou qui occupent des postes en bas de la hiérarchie professionnelle, qui ont connu des trajectoires morcelées et à temps partiel, dont de nombreuses femmes. Rosende et Schoeni (2012) ont montré que l'augmentation récente de l'emploi des seniors est principalement due à la hausse du taux d'emploi des femmes âgées de 50 et 59 ans : la part de femmes sur le marché du travail est passée de $55 \%$ en 1991 à $72 \%$ en 2010. Si les cadres supérieurs ou les femmes mariées échappent à l'impératif du travail salarié en

6 En 2009, en l'espace d'un an, seuls 2,2\% des seniors ont changé d'employeur contre $10,2 \%$ chez les $25-39$ ans et $28,3 \%$ chez les $15-24$ ans (OFS 2012).

7 Les résultats de l'enquête SHARE sont issus de l'ouvrage de De Puy, Jacqueline (2012): Travail, retraite, santé. Revue de la littérature et état des lieux. Lausanne : Fondation Charlotte Olivier.

8 La 11e révision de l'AVS (Loi sur l'Assurance vieillesse et survivants) actuellement en discussion au Parlement propose de relever l'âge légal de la retraite à 65 ans pour les femmes. 
fin de carrière, les autres se disent « coincées » : « Je n’ai pas le choix ! », « Le calcul est vite fait: moins de travail, moins d'argent et c'est le porte-monnaie qui décide... », disent-elles en entretien'. Cette nécessité économique n'est pas toujours une sinécure. L'avancée en âge s'accompagne de phénomènes d'usure au travail liés au vieillissement des corps, mais aussi aux traces laissées par les sollicitations répétées de l'activité (Kuehni et Rosende 2013). Par ailleurs, hommes et femmes décrivent, parfois avec véhémence, les transformations survenues dans leur environnement de travail : chasse aux temps morts, introduction de nouvelles technologies, évaluation individualisée des performances, intensification du travail et exigences de productivité (Molinié et al. 2012).

\subsection{Au quotidien : plus de travail et moins de soutien}

À première vue, tout semble opposer les mécaniciens et les caissières : l'environnement, la nature du travail, les conditions d'activité, la reconnaissance matérielle et symbolique puisque les uns sont des hommes qualifiés et font un métier prestigieux, alors que les autres sont majoritairement des femmes sans qualification reconnue et exercent une activité des plus dévalorisées (Ferreras 2007). Si les trajectoires professionnelles et le quotidien des uns et des autres se ressemblent peu, tous deux sont soumis à des contrôles de plus en plus stricts et connaissent des phénomènes d'intensification du travail avec l'introduction de l'automatisation nécessitant une «vigilance permanente» (Bernard 2013 : 123). La pression temporelle, comme la «traçabilité », sont extrêmement mal vécues chez les salarié.e.s interviewé.e.s. Cette situation est d'autant plus difficile à vivre qu'ils et elles ont connu « deux mondes industriels » (Veltz 2000), celui dit « classique » des Trente Glorieuses avec une organisation taylorienne et la transition vers de formes d'organisation du travail et de management davantage orientées vers la flexibilité et la responsabilité individuelle. Les interviewéee.s parlent d'une «dégradation » de leurs conditions de travail, quand le management met en avant une forme de "résistance au changement ", stéréotype largement répandu à propos des seniors (Burnay 2011). Sans être forcément privé.e.s des "repères identitaires fondamentaux» (D’Amours 2002), ces transformations amoindrissent « leur pouvoir de négociation et limite[nt] leurs possibilités de bénéficier d'un soutien organisationnel » (Zimmermann et al. 2007: 743). L'expérience acquise permet généralement aux salarié.e·s âgé.e.s de mettre en place des stratégies de préservation de la santé, notamment au sein des collectifs de travail (Molinié et Volkoff 2008), toutefois les changements bousculent passablement les modus operandi et le soutien qu'ils et elles peuvent attendre des pairs. Cumulés à l'avancée en âge, ils

9 Plusieurs femmes interviewées rapportent avoir augmenté leur taux d'occupation en seconde partie de carrière pour maximiser leur rente, notamment celles qui sont divorcées. 
contribuent donc à créer un sentiment d’insécurité chez les interviewé.e.s. Les mécaniciens redoutent un manque de concentration qui leur ferait oublier l'un des contrôles de sécurité, de plus en plus nombreux chaque année. Les caissières craignent une faute d'inattention ou une fausse manipulation et certaines espèrent être mutées en rayon pour préserver leurs membres supérieurs (épaules, poignets, coudes) des sollicitations du travail répétitif. La menace de la "faute professionnelle» pèse sur l'ensemble des salarié.e.s, toutefois la « fatigue », terme générique couramment utilisé par les interviewé.e.s pour décrire les effets de l'avancée en âge, combinée aux changements technologiques et organisationnels, crée un certain sentiment de vulnérabilité chez les seniors.

\section{Caissières et mécaniciens en seconde partie de carrière : des répertoires de résistance différenciés}

Chaque individu réagit différemment devant le travail et ses contraintes, il est toutefois possible de relever des "répertoires de résistance » totalement différenciés entre les deux groupes professionnels investigués. Développée par Charles Tilly, la notion de répertoire permet de montrer que le « choix » opéré par les individus dans les stratégies qu'ils mobilisent pour marquer leur désapprobation n'est pas le fruit d'une réflexion individuelle, mais « le résultat et le produit des structures d'interactions » (Offerlé 2008 : 183). Bélanger et Thuderoz (2010 : 428) préfèrent la notion d'opposition à celle de résistance, puisqu'elle permet de rendre compte à la fois d'une contestation (se dresser contre), mais aussi d'une continuation de la relation entre les salarié.e.s et le management puisqu'il faut "s'imposer" et "proposer une autre manière de s'organiser ». Davantage encore si l'on considère ce double mouvement, il semble nécessaire de tenir compte des ressources inégales dont disposent les caissières et les mécaniciens en seconde partie de carrière.

Avant même d'avoir accès à la parole des travailleurs/euses, nous avons pu mesurer l'écart entre ces deux groupes professionnels dans la manière dont ils sont considéré.e.s par leur hiérarchie et le statut différencié dont ils jouissent dans l'entreprise ${ }^{10}$. Les mécaniciens âgés nous ont été présentés comme des "grincheux " par leurs responsables : ils se cramponnent à leurs privilèges et "râlent à longueur de journée » exprimant leurs griefs sur «Le mur des Lamentations", tableau de communication dans le local commun où les professionnels viennent "prendre leur tour ", s'informer des derniers changements horaires, etc. Les mécaniciens sont considérés comme les «barons du rail » et jouissent d'un prestige tant dans l'entreprise qu'en dehors de

10 Les négociations qui précèdent l'enquête sont un matériau utile à l'analyse, car elles permettent souvent d'éclairer les rapports de force qui structurent le champ (Darmon 2005). 
celle-ci. La manière dont les gérants de magasin nous ont présenté les caissières est tout autre: définies comme la "crème des salariées", elles sont des employées "sur qui l'on peut compter», serviables, pour ne pas dire corvéables à merci. Si les RH de l'entreprise des transports cherchent à limiter au maximum les échanges entre les générations, car les plus « vieux ont une mauvaise influence sur les plus jeunes »; du côté de la grande distribution, la hiérarchie favorise les contacts afin de faciliter un apprentissage sur le tas et de développer « l'esprit familial » encouragé dans les magasins, même de très grande taille. Une telle opposition peut sembler caricaturale, elle n'en est pas moins opérante. Si le pouvoir de résister frontalement est inégalement partagé, il convient de l'articuler aux situations de travail et aux parcours professionnels. Par ailleurs, pour éviter de reproduire le discours managérial et entériner le fait que les uns refusent la domination quand les autres se soumettent passivement, il est nécessaire de rappeler que les résistances sont loin d'être toujours contestataires et peuvent parfois prendre des formes plus silencieuses.

\subsection{Enjeux et règles du jeu : parcours professionnels et soutiens collectifs}

Si les personnes interviewées évitent les stratégies d'exit (Hirschman 1970) dans la mesure où elles veulent s'assurer une retraite décente, hommes et femmes ne jouent pas dans les mêmes «catégories ». D’une part, le montant des rentes en jeu n'est pas comparable : les conditions de rémunération vont du simple au double et la durée de cotisation varie considérablement selon le sexe. Les mécaniciens ont cotisé durant l'ensemble de leur vie active sur ce qu'ils définissent comme un «bon salaire » et à plein temps ; alors qu'une majorité de caissières ont ce qu'elles appellent des «trous de cotisation" suite aux interruptions volontaires ou involontaires dans leur parcours professionnel et ont souvent travaillé à temps partiel pour des salaires relativement bas ${ }^{11}$. D'autre part, l'enjeu financier du maintien en emploi recouvre des réalités pour le moins disparates. Pour les mécaniciens, il s'agit avant tout de maintenir un niveau de vie antérieur, alors que pour les caissières il s'agit souvent d'éviter une situation de dépendance, à l'égard d'un conjoint pour celles qui sont mariées, ou de l'État pour celles qui vivent seules et risquent de connaître la pauvretéé12.

11 Le système de retraite helvétique, fondé sur le modèle du pourvoyeur principal, désavantage clairement les femmes du fait de la division sexuelle du travail et de leur assignation prioritaire à la sphère reproductive (Kuehni et al. 2013). Cette inégalité est clairement repérable si l'on tient compte du type de rente par sexe et le montant des celles-ci (OFS, 2011).

12 En 2010, 8,4\% des hommes de plus de 65 ans contre 14,5\% de femmes touchent des prestations complémentaires, car leur retraite est insuffisante, par ailleurs les enquêtes soulignent d'importants phénomènes de « pauvreté cachée » (OFS, 2011). 
«Je ne sais pas combien je vais toucher, cela m'angoisse tellement que je préfère faire l'autruche. (...) Je ne sais même pas si je vais pouvoir payer mon loyer! » (Caissière, 61 ans, divorcée, 17 ans d'ancienneté, a augmenté son taux d'occupation à $100 \%$ dès l'âge de 55 ans).

Si les femmes âgées apparaissent comme moins revendicatrices que les hommes, ce n'est pas seulement parce qu'elles ont beaucoup à perdre, mais c'est aussi parce qu'elles sont moins bien protégées. La contestation ouverte n'est jamais exempte de risques, notamment en Suisse où il n'existe aucune protection contre le licenciement. Les mécaniciens bénéficient de nombreux soutiens, inexistants chez les caissières. Le domaine du rail s'est constitué autour d'un «bastion masculin » fortement syndiqué, qui a longtemps réuni des ouvriers dépositaires d'une «identité de métier » (Zarca 1988). Les mécaniciens sont aujourd'hui encore organisés sur le modèle de la corporation et jouissent d'un pouvoir extrêmement développé dans l'entreprise. Près de $100 \%$ des travailleurs sont syndiqués et cela les protège sur un double plan : d'une part au niveau collectif, le syndicat joue un rôle de contrepoids face aux positions patronales et, d'autre part au niveau individuel, c'est une sorte d'assurance qui permet de dire non aux heures supplémentaires ou à la nouvelle grille salariale sans risquer une mise à pied.

En revanche, les caissières sont peu organisées collectivement et sont faiblement syndiquées. Dans le commerce de détail, le taux de syndicalisation est estimé à $10 \%$ toutes professions confondues (Unia 2012). La concurrence est très développée dans ce secteur d'activité : salaire au mérite, pressions sur les horaires, accès différencié au plein temps et réserve de main d'œuvre contribuent fortement à la construction d'un rapport individualisé au travail et limitent les possibilités de résistances collectives. Ferreras (2007 : 127) met en avant un « rapport à autrui domestiqué » largement entretenu par un « régime de faveur » mis en place dans les magasins : l'entraide est répandue, en revanche l'action politique collective est difficile ${ }^{13}$. Les caissières interviewées mettent un point d'honneur à « s'occuper de leurs affaires » ou «balayer devant leur porte » et sont avant tout préoccupées par le fait de se maintenir en emploi et de négocier les conditions de ce maintien avec leur hiérarchie directe. Certaines d'entre elles ont réussi à accéder à un poste en rayon, réputé moins pénible physiquement, "grâce aux bonnes relations avec leur responsable », mais elles sont loin de revendiquer des adaptations de postes pour l'ensemble des salariées âgées.

\subsection{Travailleurs et travailleuses inégaux face au « vieillissement actif »}

Les responsables RH des entreprises helvétiques commencent à se soucier du renouvellement des générations et du transfert des connaissances, toutefois les dispositifs

13 Il est important de souligner qu'il ne s'agit pas d'une incapacité à l'action collective (Benquet 2009). 
de "gestion prévisionnelle des âges » sont à ce jour peu formalisés et destinés à un nombre restreint de salarié.e.s (Le Feuvre et al., 2014). Les entreprises souhaitent limiter les départs précoces à la retraite de leur personnel qualifié, technique ou d'encadrement (principalement des hommes), mais se montrent beaucoup plus ambivalentes à l'égard des investissements à consentir en matière de formation continue et/ou d'adaptation des postes de travail en faveur du personnel moins qualifié et facilement «remplaçable », dont de nombreuses femmes. Les RH (tout comme les politiques) cherchent à éviter "une pénurie de salarié.e.s qualifié.e.s », laissant dans l'ombre les seniors qui occupent des positions subalternes dans la hiérarchie socioprofessionnelle. La qualification joue donc clairement comme une ressource pour la contestation et ce, d'autant plus quand il s'agit de faire reconnaître la pénibilité du travail et l'articuler aux effets du vieillissement. Si les mécaniciens s'opposent collectivement aux nouvelles grilles horaires, dénoncent la pénibilité du travail et revendiquent que les plus âgés puissent être replacés dans l'entreprise lorsqu'ils ont des difficultés à tenir le poste, les caissières disposent de peu de relais pour faire entendre leurs difficultés dans l'exercice de leur travail. Les arguments avancés par les premiers portent non seulement sur la pénibilité du métier, mais mobilisent également les clients : il s'agit de garantir un service public de qualité et assurer la sécurité des passagers.

- «lls ne pourront pas nous faire conduire jusqu'à septante ans, faut être réaliste ! C'est dangereux, faut pas oublier qu'on fait un métier à risques...

- Pour vous?

- Oui, mais aussi les pour les clients, (...) il ne fait pas bon monter dans un train avec un gaillard trop vieux à l'avant ! Et puis, ce n'est pas très bon pour l'image... »

(Mécanicien, 64 ans, 30 ans d'ancienneté, travaille à $100 \%$ )

Les caissières sont tout aussi soucieuses que les mécaniciens de la qualité du travail fourni et cherchent également à préserver leur santé, elles n'ont cependant par les mêmes arguments, ni les mêmes ressources pour faire entendre leur « voix ». La situation des caissières en seconde partie de carrière se définit largement par une forme d'«invisibilité » qui caractérise plus largement l'ensemble des travailleuses âgées (Ainsworth 2002).

\subsection{Résistances invisibles : I'apport d'une perspective de genre}

Si les caissières ne s'opposent pas frontalement aux heures supplémentaires, au morcellement de leur horaire ou à leur niveau de salaire, ce n'est pas pour autant qu'elles consentent aux rapports d'exploitation et de domination. Au regard des risques qu'entraîne toute manifestation de contestation ouverte pour les caissières, leur silence peut certes revêtir les signes de la soumission, mais il n'est pas pour autant la "marque de l'intériorisation d'une indignité consentie»(Coste et al. 2008: 20). L'absence de parole collective sur l'oppression au travail ne signifie pas une absence 
de "pratiques de résistance » (Avril et al. 2010). Plusieurs enquêtes ont mis au jour les stratégies individuelles, plus rarement collectives, pour se réapproprier leur travail : ralentissements, vols, sabotages, tactiques pour répondre aux clients maltraitants, etc. (Barbier 2012 ; Waelli et Fache 2011). Si notre dispositif d'enquête ne permettait pas d'observer des pratiques de résistances au travail, nombreuses sont les caissières à avoir mentionné le fait «d'aller à leur rythme», d'avoir mis en place des stratégies pour répondre aux clients insupportables ou encore de s'arranger entre collègues pour trouver des moments de pause loin du "rush des caisses», par exemple en se rendant dans les rayons pour remettre une marchandise.

Discutant le modèle de Hirschman, Bajoit (1988) a déjà relevé que le fait de quitter une situation insatisfaisante ou un lieu tel que l'entreprise (exit) ou de la contester ouvertement (voice) n'est pas le seul modèle de résistance possible. Pour l'auteur, il existe différentes manières d'être «loyal» : soit en participant activement à la relation, ce qu'il nomme loyauté (loyalty), soit passivement, ce qu'il nomme apathie ${ }^{14}$. L'apathie permet de mettre au jour des comportements de salariée.s qui ne contestent pas ouvertement la légitimité du pouvoir, mais de manière souterraine. L'apathie, à l'instar de la loyauté, n'ouvre pas sur le conflit et contribue également à reproduire le contrôle social, bien qu'elle provoque une détérioration de la coopération. Les salarié.e.s apathiques profitent en quelque sorte de leur statut, et en font le moins possible. Toutefois, la notion d'apathie rend assez mal compte de la situation des caissières en seconde partie de carrière (et plus généralement de la situation des femmes au travail15). Tout d'abord parce qu'elle est porteuse d'une connotation négative et dénote une absence d'énergie ou une certaine mollesse, mais aussi parce que l'apathie ne permet pas de prendre la mesure des enjeux qui structurent l'engagement au travail. La volonté des caissières de conserver leur emploi pour constituer une retraite décente, mais surtout leur attachement à leur travail rémunéré, sont les moteurs nécessaires pour investir quotidiennement la caisse d'un grand magasin. La reconnaissance d'un droit au travail salarié a été durement acquise pour certaines d'entre elles; aussi, si elles ne contestent pas ouvertement leur niveau de salaire et disent passer par-dessus leur sentiment de fatigue ou leurs douleurs articulaires, c'est parce que les enjeux qui structurent leur engagement au travail ne se comprennent qu'à la lumière des différents rapports de domination qu'elles subissent.

14 Pour Bajoit (1988 : 328), « il est évident que, si les uns sont fidèles par conviction, par attachement au système d'interaction, à ses dirigeants, à ses objectifs, les autres ne désertent ni ne protestent que par un mélange de résignation, de passivité, de repli sur soi, que j'appelle apathie ».

15 Pour une discussion plus large sur le sujet, se référer à Morgane Kuehni (2011), particulièrement le chapitre IV. 


\section{Conclusion}

Les grèves, pour prendre l'exemple prototypique d'une contestation ouverte, ne sont pas uniquement mises en place par des hommes qualifiés et syndiqués. Les travailleuses, mais aussi celles et ceux que l'on désigne aujourd'hui comme les "précaires ", opposent parfois une résistance frontale et organisée. Il s'agit cependant de «mobilisations improbables » (Benquet 2009) dans la mesure où les conditions de travail et les parcours de vie rendent leur émergence difficile. Mobiliser une réflexion sur les conditions de travail et les trajectoires de vie permet d'éviter une lecture univoque des résistances au travail. En seconde partie de carrière, les caissières et les mécaniciens doivent faire face à un enjeu identique : tenir le plus longtemps possible dans une situation de travail qui pose différents problèmes compte tenu de l'avancée en âge. Pourtant ce qui est " en jeu », comme les «règles du jeu » varient considérablement et expliquent la pluralité des registres de contestation mobilisés. La contrainte au maintien en emploi n'a ni le même poids, ni ne recouvre les mêmes problématiques, et conditionne largement les possibilités de contestation puisque les unes et les autres ne disposent pas des mêmes ressources pour faire entendre leur voix dans les entreprises. Si les mobilisations «classiques » du travail ont longtemps été sur le devant des scènes publique et scientifique, il convient de reconsidérer les outils théoriques et méthodologiques pour penser les résistances des salariées. Comme l'écrit très justement Emmanuelle Lada (2008: 183), il est moins question « d'invisibilité que d'invisibilisation " des pratiques de résistances féminines, habituellement occultées en raison d'une grille de lecture inadéquate ou de la conception que se font les chercheurs/euses de ce que sont, ou devraient être, les résistances au travail. Certes, les pratiques clandestines ne renversent pas l'ordre établi, mais aussi discrètes soientelles, elles « contestent en contournant, (et) contraignent ceux qui régentent et tirent profit de l'ordre à rechercher des ajustements » (Jounin 2008 : 43).

\section{Bibliographie}

Ainsworth S. (2002) : "The Feminine "Advantage": A Discursive Analysis of the Invisibility of Older Women Workers ». Gender, Work and Organization, vol. $9 \mathrm{n}^{\circ} 5$, pp. 579-601.

Avril C., Cartier M. et Serre D. (2010) : Enquêter sur le travail. Concepts, méthodes, récits. Paris : La Découverte.

Bajoit G. (1988) : «Exit, voice, loyalty... and apathy. Les réactions individuelles au mécontentement ». Revue française de sociologie, vol. $29 \mathrm{n}^{\circ} 2$, pp. 325-345.

Barbier P. (2012) : «Contrainte relationnelle et résistance au travail. Les vendeurs des grands magasins ». Sociétés contemporaines, vol. 86 n², pp. 31-57. 
Bélanger J. et Thuderoz C. (2010) : «Le répertoire de l'opposition au travail ». Revue française de sociologie, vol $51 \mathrm{n}^{\circ} 3$, pp. 427-460.

Benquet M. (2009) : "Les raisons de l'action collective : retour sur la mobilisation improbable des salariés d'hypermarchés». Sociologie du travail, vol. 52 n³, pp. 305-322.

Bernard S. (2013) : «Travailler “à l'insu” des clients. Défaut de reconnaissance en caisses automatiques ». Travailler, vol. 29 n¹, pp. 119-139.

Bouffartigue P. (2008): "Précarités professionnelles et action collective. La forme syndicale à l'épreuve ». Travail et Emploi, n¹16, pp. 33-43.

Bouquin S. (2011) : « La question des résistances au travail dans la sociologie du travail française ». Actuel Marx, n49, pp. 60-72.

Bouquin S. (Ed.) (2008) : Résistances au travail. Paris : Syllepse.

Burnay N. (2011): «La cohabitation intergénérationnelle en entreprise: des stéréotypes prégnants à l'égard des seniors ». Communication et organisation, $\mathrm{n}^{\circ} 40$, pp. 71-86.

Castel R. (2009): La montée des incertitudes. Travail, protection, statut de l'individu. Paris : Seuil.

Coste F., Costey P. et Tangy L. (2008) : «Consentir : domination, consentement et déni ». Tracés. Revue de Sciences humaines, n¹4, [En ligne], consulté le 12 mai 2013, http:// traces.revues.org/365.

D’Amours M. (2002) : Diversification et fragmentation du travail. Le passage de l'emploi salarié typique à des formes de travail atypique chez les travailleurs de plus de 45 ans. Montréal : INRS-Urbanisation, Culture et Société.

Darmon M. (2005) : « Le psychiatre, la sociologue et la boulangère : analyse d'un refus de terrain ». Genèses, n58, pp. 8-12.

De Puy J. (2012) : Travail, retraite, santé. Revue de la littérature et état des lieux. Lausanne: Fondation Charlotte Olivier.

Ferreras I. (2007) : Critique politique du travail. Travailler à l'heure des services. Paris: Presses de la Fondation nationale des sciences politiques.

Guillemard A.-M. (2003) : L'âge de l'emploi. Les sociétés face à l'épreuve du vieillissement. Paris : Armand Colin.

Hirschman A. (1970): Exit, Voice and Loyalty : Responses to Decline in Firms, Organizations, and States. Cambridge, Mass : Harvard University Press. 
Jounin N. (2008) : «Humiliations ordinaires et contestations silencieuses. La situation des travailleurs précaires des chantiers ». Sociétés Contemporaines, n70, pp. 25-43.

Kuehni M. (2011) : Le travail des sans-emploi : analyse sociologique de l'assignation à un programme d'emploi temporaire. Thèse de doctorat, Université de Lausanne.

Kuehni M. et Rosende M. (2013) : «Inégalités de santé au travail chez les seniors: l'apport du genre ». Revue économique et sociale, vol.71 n²2, pp. 57-64.

Kuehni M., M. Rosende et Schoeni C. (2013) : "Maintien en emploi et inégalités de sexe ». Lien social et politiques, $\mathrm{n}^{\circ} 69$, pp. 197-213.

Le Feuvre N., Kuehni M., Rosende M. et Schoeni C. (2014): «Le genre du "vieillissement actif" : du principe du traitement équitable à la multiplication des injonctions contradictoires ». Revue suisse de sociologie, vol. $40 \mathrm{n}^{\circ} 2$, in press.

Molinié A.-F., Gaudart C. et Pueyo V. (Ed.) (2012) : La vie professionnelle. Âge, expérience et santé à l'épreuve des conditions de travail. Toulouse : Octarès.

Molinié A.-F. et Volkoff S. (2010) : «Santé au travail : jusqu'où se logent les inégalités sociales ? Entretien réalisé par Emmanuelle Cambois ». Retraite et société, $\mathrm{n}^{\circ} 59$, pp. 164-173.

OCDE (2006) : Vivre et travailler plus longtemps. Paris : OCDE.

Offerlé M. (2008) : «Retour critique sur les répertoires de l'action collective (XVIII XXI ${ }^{\mathrm{e}}$ siècles) ». Politix, vol. 81 n¹, pp. 181-202.

OFS (2012) : DEMOS : Newsletter informations démographiques, $\mathrm{n}^{\circ} 2$, mai.

OFS (2011): Indicateurs de la prévoyance vieillesse. Résultats des indicateurs clés. Neuchâtel : OFS.

Puech I. (2004) : « Le temps du remue-ménage. Conditions d'emploi et de travail des femmes de chambres ». Sociologie du travail, vol. 46 n², pp. 150-167.

Rosende M. et Schoeni C. (2012) : Seconde partie de carrière, régime de retraite et inégalités de sexe. Revue française des affaires sociales, vol. 2 n²-3, pp. 130-147.

Rot G. (2000) : « La résistance ouvrière face aux nouvelles formes de rationalisation: entre restriction et résurgence ». In Actes du colloque GREPO, $n^{\circ}$ spécial de la revue Travail, emploi, formation, $\mathrm{n}^{\circ} 1$, pp. 13-29.

Thompson P. et Ackroyd S. (1995) : « All quiet at the workplace front ? A critique of recent trends in British industrial sociology ». Sociology, vol. 29 n4, pp. 615-633.

Unia (2012) : Branche du commerce de détail. Analyse, objectifs et revendications d'Unia. Berne : Syndicat Unia.

Veltz P. (2000) : Le nouveau monde industriel. Paris : Gallimard. 
Zarca B. (1988): «Identité de métier et identité artisanale». Revue française de sociologie, vol. $29 \mathrm{n}^{\circ} 2$, pp. 247-273.

Zarifian Ph. (2004) : «Travail, modulation et puissance d'action ». L'Homme et la société, $\mathrm{n}^{\circ} 152-153$, pp. 201-227.

Zimmermann H., Fournier G. et Gauthier C., (2007): "Analyse des conduites de salariés en transition de fin de carrière: le cas de travailleurs et travailleuses en situation d'emploi atypique ». Relations industrielles, vol. 62 n4, pp. 740-767.

Waelli M. et Fache Ph. (2011) : «Quand le sujet “encaisse”. À propos du détournement des règles de travail en hypermarchés ». Revue internationale de psychosociologie et de gestion des comportements, $\mathrm{n}^{\circ} 43$ vol. XVII, pp. 149-158. 